\title{
INFLUÊNCIA DO TRATAMENTO COM ETILENO SOBRE O TEOR DE SÓlIDOS SOLÚVEIS E A COR DE PIMENTAS $\left({ }^{1}\right)$
}

\author{
GISELDA MARIA PEREIRA $\left({ }^{2} *\right)$; FERNANDO LUIZ FINGER $\left({ }^{2}\right)$; VICENTE WAGNER DIAS CASALI $\left({ }^{2}\right)$; \\ SÉRGIO HERMÍNIO BROMMONSCHENKEL ( $\left.{ }^{3}\right)$
}

\begin{abstract}
RESUMO
Este trabalho teve como objetivo avaliar a variabilidade genética em frutos de Capsicum com relação ao acúmulo de sólidos solúveis totais, degradação de clorofila, síntese de carotenóides e tempo necessário para o completo amadurecimento de frutos, em resposta ao tratamento com ethephon. O tratamento com ethephon provocou alteração do conteúdo de sólidos solúveis totais em frutos dos acessos BGH 4366 (C. baccatum) e BGH 4708 (C. frutescens), enquanto os demais acessos não foram responsivos ao tratamento. O amadurecimento dos frutos foi caracterizado por decréscimo no conteúdo de clorofila e conteúdo de carotenóides, contudo, não foi detectada variabilidade genética entre os acessos para a degradação da clorofila e para a síntese de carotenóides, quando tratados com ethephon. O uso de ethephon acelerou o amadurecimento nos acessos BGH 4179 (C. frutescens), BGH 6029 (C. baccatum) e Ca 6 (C. annuum).
\end{abstract}

Palavras-chave: amadurecimento, etileno, clorofila, carotenóides.

\section{ABSTRACT \\ INFLUENCE OF ETHYLENE TREATMENT ON THE TOTAL SOLIDS CONTENT AND COLOR OF PEPPERS}

The goal of the present work was evaluate the genetic variability regarding the accumulation of total soluble solids, degradation of chlorophyll, synthesis of carotenoids and number of days to full ripening of Capsicum fruits treated with ethephon. The treatment with ethephon altered the content of soluble solids in the fruits of accessions BGH 4366 (C. baccatum) and BGH 4708 (C. frutescens), while the remaining accessions did not show any changes when treated with ethephon. The ripening was characterized by the degradation of chlorophyll and increase in the synthesis of carotenoids in all fruit, but there was no variability among the accessions when treated with ethephon. The ethephon was efficient in inducing the ripening of BGH 4179 (C. frutescens), BGH 6029 (C. baccatum) and Ca 6 (C. annuum) accessions.

Key words: ripening, ethylene, chlorophyll, carotenoids.

( $\left.{ }^{1}\right)$ Recebido para publicação em 7 de novembro de 2006 e aceito em 18 de julho de 2008.

$\left({ }^{2}\right)$ Departamento de Fitotecnia, Universidade Federal de Viçosa, 36571-000 Viçosa (MG), Brasil. E-mail: gilseldabiors@yahoo.com.br $\left({ }^{*}\right)$ Autora correspondente.

$\left({ }^{3}\right)$ Departamento de Fitopatologia, Universidade Federal de Viçosa, 36571-000 Viçosa (MG), Brasil. 


\section{INTRODUÇÃO}

O gênero Capsicum compreende tanto espécies de pimentas silvestres quanto espécies cultivadas, tais como C. annuum, C. frutescens, C. chinense, C. baccatum e C. pubescens (CAsali e Couto, 1984). As espécies pertencentes a esse gênero são originárias da América tropical e têm distribuição cosmopolita (MC LEOD et al; 1983). A popularidade das espécies de Capsicum é atribuída, principalmente, às suas qualidades organolépticas, como coloração e sabor (REIFSCHNEIDER, 2000). Devido a essas importantes características, os frutos de pimenta, verdes e maduros, são utilizados, como condimentos em diversos pratos e produtos alimentícios industriais (CASALI e CoUTO, 1984).

A utilização industrial dos frutos de pimenta requer que tenham tamanho, formato e grau de maturação uniformes, bem como, maior rendimento e coloração adequada. Visando à obtenção de produtos mais adequados para esta finalidade, os trabalhos de melhoramento genético consideram características como coloração, formato do fruto, teor de sólidos solúveis e espessura da polpa, sendo as estas duas características as principais responsáveis pelo rendimento industrial. (CASALI e STRINGHETA, 1984).

O regulador de crescimento etileno e seus análogos têm sido muito utilizados para acelerar e uniformizar o amadurecimento de frutos de diversas espécies (Abeles et al., 1992). O processo de amadurecimento de frutos compreende mudanças bioquímicas, fisiológicas e visuais, tais como alterações na taxa respiratória, na coloração, no sabor, na composição de açúcares redutores, na textura e na produção de substâncias voláteis (TUCKER, 1993). Muitas destas modificações podem estar relacionadas à síntese de etileno ou à aplicação exógena desta substância (ABELES et al., 1992). Contudo, a resposta dos frutos ao tratamento com etileno, depende da sensibilidade do tecido, da concentração utilizada, da composição da atmosfera, do tempo de exposição, da temperatura e da sensibilidade da espécie ou cultivar (SaltVeit, 1999).

Em pimentas, grande número de estudos tem sido realizado sobre os efeitos do etileno exógeno ou de seus análogos na síntese e degradação de pigmentos (MAO e MotSEnBocker, 2002; KRAJAYKLANG et al., 1999; Pretel et al., 1995; Saltveit Jr, 1977), na pungência e na velocidade de amadurecimento (KRAJAYKLANG et al., 1999), na da taxa respiratória e na síntese de etileno pelo fruto durante o amadurecimento (TADESSE et al., 2002; VILLAVINCENCIO et al., 2001; SALtVEIT Jr, 1977).

O objetivo deste trabalho foi avaliar e variabilidade genética associada ao acúmulo de sólidos solúveis totais, à degradação de clorofila à síntese de carotenóides no amadurecimento póscolheita de frutos de Capsicum, em resposta à aplicação de ethephon.

\section{MATERIAL E MÉTODOS}

Foram estudados 10 acessos das espécies $C$. baccatum, C. chinense, $C$. frutescens e $C$. annuum, sendo os acessos das três primeiras espécies provenientes do Banco de Germoplasma de Hortaliças da Universidade Federal de Viçosa e os acessos da última, oriundos da Universidade do Estado do Novo México (New Mexico State University).

A semeadura foi realizada em bandejas de isopor com substrato comercial. Após 60 dias, as mudas foram transplantadas para vasos de $12 \mathrm{~L}$, mantendo-se uma planta por vaso, em casa de vegetação. As plantas foram dispostas inteiramente ao acaso, com quatro repetições. O cultivo foi realizado no período de junho de 2003 a janeiro de 2004 .

Para as análises do teor de sólidos solúveis totais e da síntese e degradação de pigmentos foram utilizados quatro tratamentos: frutos verdes, frutos amadurecidos na planta e frutos amadurecidos fora da planta, tratados ou não com ethephon, cujo componente ativo ácido 2-cloroetilfosfônico libera etileno quando absorvido pela célula, com o total de quatro repetições. Os frutos verdes e amadurecidos na planta foram colhidos e armazenados a $-20^{\circ} \mathrm{C}$. Para o estudo dos efeitos do ethephon, os frutos foram colhidos no estádio verde-maduro, e após a desinfecção com solução de hipoclorito de sódio a $0,5 \%$, foram separados em dois grupos: os tratados com ethephon e os frutos-controle não-tratados com ethephon. Para o tratamento, os frutos foram mergulhados em solução de ethephon $1000 \mathrm{mg} \mathrm{L}^{-1}$, por 30 minutos. Frutos tratados e não tratados foram armazenados em câmara a $22{ }^{\circ} \mathrm{C}$ e umidade relativa de $80 \% \pm 5$ até atingirem o completo amadurecimento.

O teor de sólidos solúveis totais da polpa de quatro frutos foi avaliado em refratômetro da marca Abbe (ATAGO 3T, Japão), após descongelamento dos frutos e maceração em almofariz, no momento da análise. Os valores correspondentes ao total de sólidos solúveis ( ${ }^{\circ}$ Brix) foram corrigidos para $20^{\circ} \mathrm{C}$, conforme método desenvolvido no InSTITUTO Adolfo Lutz (1985).

As alterações nos teores de clorofila e pigmentos carotenóides foram medidas de acordo com método proposto por Hendry e Price (1993), modificado pelo emprego de um grama de tecido do fruto para cada $25 \mathrm{~mL}$ de solução de acetona amoniacal. Após a maceração, o extrato foi filtrado e 
os teores de clorofila e pigmentos carotenóides determinados por leitura em espectrofotômetro.

O número de dias necessários para o completo amadurecimento foi definido para frutos tratados com ethephon e controles, com quatro repetições, mantidos nas condições anteriormente descritas, desde o dia da colheita até atingirem o estádio $100 \%$ vermelho ou amarelo maduro.

\section{RESULTADOS E DISCUSSÃO}

\section{Teores de sólidos solúveis totais}

Foram observados teores maiores de sólidos solúveis totais em frutos amadurecidos na planta do que em frutos verde-maduros em todos os acessos avaliados, conforme indica a tabela 1 . O aumento observado na concentração de sólidos solúveis totais durante o amadurecimento está de acordo com o que foi observado por NIELSEN et al. (1991) e por TADESSE et al.(2002), que estudaram cultivares de (С. апnиит).

Comparações entre os frutos verde-maduros e frutos colhidos verdes e amadurecidos fora da planta que não foram tratados com ethephon, não revelaram diferenças significativas entre o teor de sólidos solúveis totais, em acessos de C. baccatum - BGH 1739 e BGH 4366, C. frutescens - BGH 4708 e C. annuum Ca 6 e Ca 13 (Tabela 1). Contudo, como mostra a tabela 1 , o tratamento com ethephon contribuiu para maior acúmulo de sólidos solúveis nos frutos desses acessos e conseqüentemente estes frutos podem ter maior rendimento industrial.

Considerando-se frutos colhidos verdes e amadurecidos fora da planta, os controles e os tratados com ethephon, observou-se que o tratamento induziu maior acúmulo de sólidos solúveis totais dos acessos BGH 4366 (C. baccatum) e BGH 4708 (C. frutescens). Este fato comprova que estes acessos responderam de forma positiva ao tratamento com ethephon e este pode implicar maior rendimento industrial aos frutos.

\section{Degradação de clorofila e síntese de carotenóides}

A análise dos teores de clorofila total nos frutos verde-maduros e amadurecidos na planta evidenciou decréscimos significativos em frutos maduros de todos os acessos, independentemente do tratamento, segundo a tabela 2. Resultados semelhantes foram obtidos por PRETEL et al. (1995), em estudos com pimentão (C. annuum). Na comparação entre frutos colhidos verdes e amadurecidos fora da planta, na presença ou na ausência de ethephon, constatou-se que o tratamento com este produto não gerou aumento significativo na degradação de clorofila em nenhum dos acessos conforme a tabela

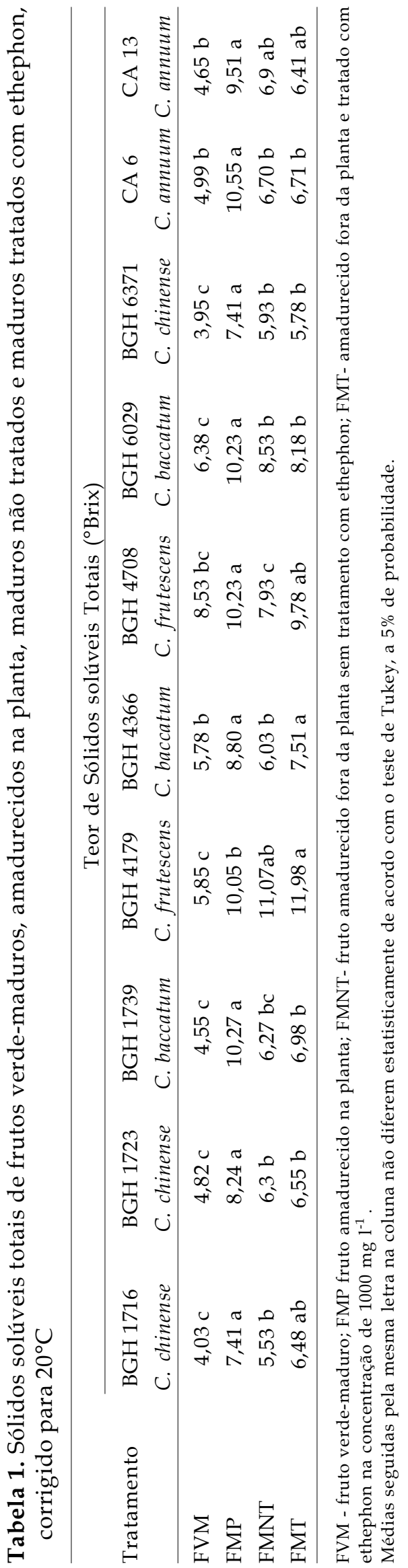

Bragantia, Campinas, v.67, n.4, p.1031-1036, 2008 

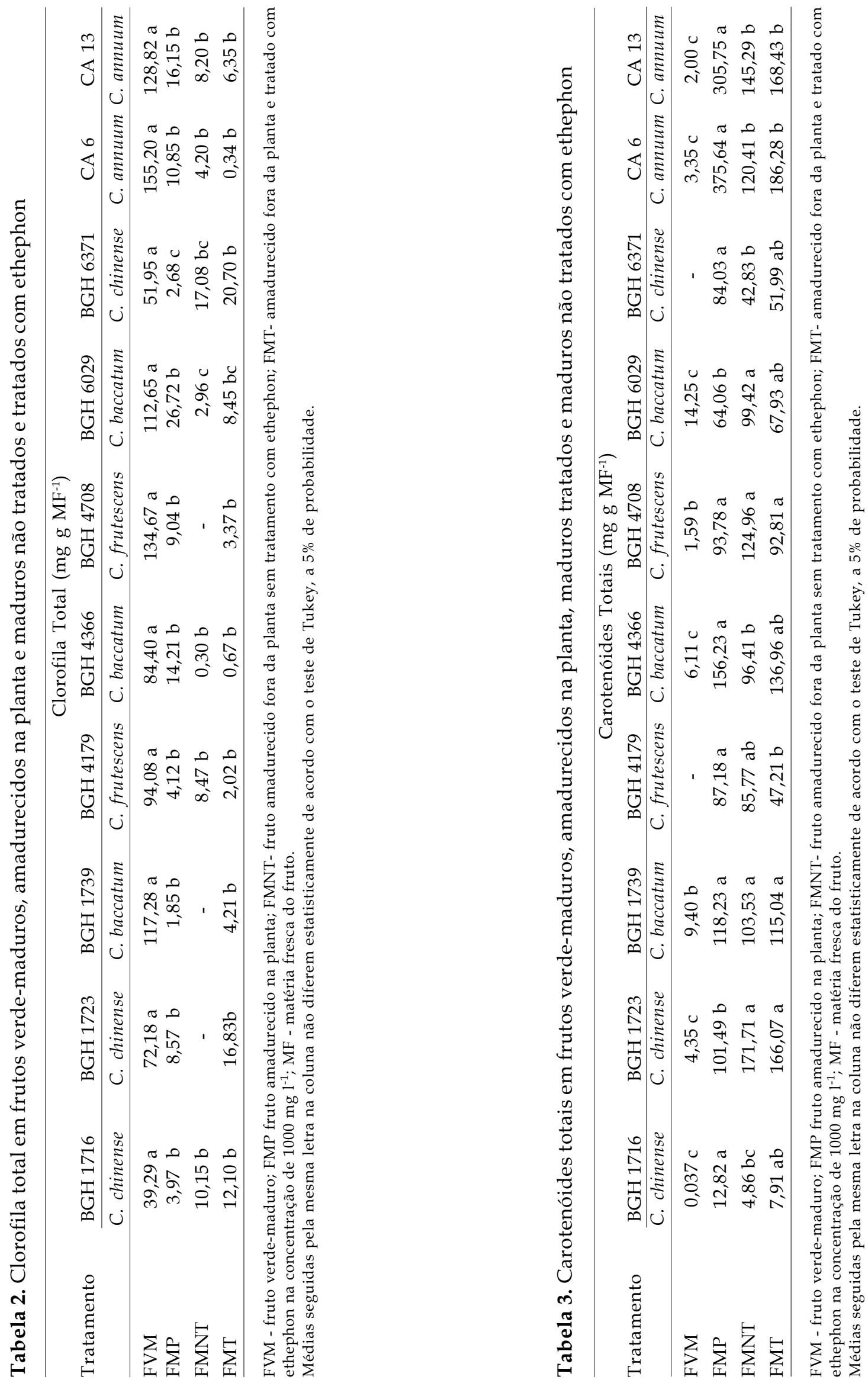
2. Este fato pode estar relacionado à insensibilidade de algumas espécies do gênero Capsicum ao etileno para esta característica.

No que se refere à síntese de pigmentos carotenóides, ao se compararem frutos verde-maduros e amadurecidos na planta, observou-se que houve aumento na síntese de carotenóides totais durante o amadurecimento para todos os acessos, conforme a tabela 3. Contudo, pela análise dos teores de carotenóides totais, em frutos colhidos verdes e amadurecidos na ausência ou na presença de ethephon, verificou-se que os últimos não continham teores superiores de carotenóides, ressaltando que a síntese ocorreu independentemente da aplicação de etileno exógeno. Portanto, o ethephon não foi efetivo em promover aumento nos teores de carotenóides sintetizados pelos frutos ao final do amadurecimento. Contudo, segundo LeLIÉvE et al. (1997), a síntese de carotenóides pode ser etileno-dependente ou independente, em função do tipo de pigmento e, portanto, outras substâncias reguladoras podem interferir nesse mecanismo. Além disso, as mudanças na coloração em frutos são afetadas pela ação do etileno (LARRIEGAUDIERE et al., 1996) e pela idade do fruto na hora da colheita (AgAr et al.,1999), sendo, muitas vezes, associadas à produção de etileno pelos frutos (ABDi et al. 1997).

\section{Antecipação do amadurecimento}

A aplicação de ethephon antecipou o amadurecimento dos frutos dos acessos BGH 4179 (C. frutescens), BGH 6029 (C. baccatum) e Ca 6 (C.annuum) e para os demais acessos, não houve efeito do tratamento com este produto segundo a tabela 4 . Resultados semelhantes foram observados por PRETEL et al. (1995), em que o ethephon não foi efetivo em acelerar o amadurecimento de frutos da variedade Califórnia, pertencente à espécie C. annuum. Contudo, a antecipação do amadurecimento devido ao tratamento com ethephon foi observada em frutos de $C$. frutescens (MAO e MOTSENBOCKER, 2002), em pimentão (C. annuum) (LocKWood e VINES, 1972), em pimentas tipo páprica e em pimentas Cayene (KRAJAYKLANG et al., 1999) ambas pertencentes à espécie $C$. annuum. Diante dos resultados, pode-se afirmar que o efeito do ethephon em acelerar o amadurecimento de pimentas variou nos diferentes acessos.

\section{CONCLUSÕES}

1. Nos acessos BGH 4366 (C. baccatum) e BGH 4708 (C. frutescens), o tratamento com ethephon provocou aumento no teor de sólidos solúveis totais dos frutos comprovando que este produto pode ser utilizado para amadurecer os frutos fora da planta com possível aumento no rendimento industrial. 
2. Não houve envolvimento do ethephon na síntese e degradação de pigmentos em nenhum dos acessos, portanto sua utilização não é recomendada para obtenção de frutos maduros com coloração mais intensa.

4. A aplicação de ethephon acelerou o completo amadurecimento apenas nos acessos BGH 4179 (C. frutescens), BGH 6029 (C. baccatum) e Ca 6 (C. апnиит), logo apenas para esses acessos é viável sua aplicação para a antecipação do amadurecimento.

5. Foi possível observar resposta diferencial ao tratamento com ethephon, visto que existe variabilidade inter e intra-específica na resposta a este regulador de crescimento.

\section{AGRADECIMENTOS}

Ao CNPq pela concessão da bolsa de mestrado a Giselda Maria Pereira e à FAPEMIG pelo auxílio financeiro para a realização da pesquisa.

\section{REFERÊNCIAS}

ABDI, N.; HOLFORD, P.; MCGLASSON, W.B.; MIZRAHI, Y. Ripening behavior and responses to propilene in four cultivars of japanese type plums. Postharvest Biology and Technology, Amsterdam, v. 12, p. 21-34, 1997.

ABELES, F. B.; MORGAN, P.W.; SALTVEIT JR, M. E. Ethylene in Plant Biology. 2.ed. São Diego: Academic Press, 1992. 414p.

AGAR, I.T.; BIASI, W.V.; MITCHAM, E.J. Exogenous ethylene accelerates ripening responses in Bartlett pears regardless of maturity or growing region. Postharvest Biology and Technology, Amsterdam, v. 17, p. 67-78, 1999.

BOSLAND, P. W.; VOTAVA, E. J. Peppers: vegetable and Spice Capsicums. CABI Publishing, 1999. 204p.

CASALI, V.W.D.; COUTO, F.A. A. Origem e botânica de Capsicum. Informe Agropecuário, Belo Horizonte, v.10, p. 8-10, 1984.

CASALI, V.W.D.; STRINGHETA, P.C. Melhoramento de pimentão e pimenta para fins industriais. Informe Agropecuário, Belo Horizonte, v.10, p. 23-25, 1984.

HENDRY, G. A. F.; PRICE, A. H., Stress indicators: chlorophylls and carotenoids. IN: HENDRY, G. A. F.; GRIME, J. P. Methods in Comparative Plant Ecology, a laboratory manual. London: Chapman e Hall, p.148-152. 1993.

INSTITUTO ADOLFO LUTZ. Normas Analíticas do Instituto Adolfo Lutz: Métodos Químicos e Físicos para Análise de Alimentos. São Paulo, 1985. v.1, 533p.

KRAJAYKLANG, M.; KLIEBER, A.; WILLS, R. B. H.; DRY, P. R. Effects of ethephon on fruit yield, colour and pungency of Cayenne and Paprika peppers. Australian Journal of Experimental Agriculture, Collingwood, v. 39, p. 81-86, 1999.
LARRIEGAUDIERE, C.; PINTO, E.; VENDRELL, M. Differential effects of ethephon and seniphos on color development of 'Starking Delicious' Apple. Journal of the American Society for Horticultural Science, Alexandria, v. 121, p. 746-750, 1996.

LELIÈVE, J.; LATCHÉ, A.; JONES, B.; BOUZAYEN, M.; PECH, J. Ethylene and fruit ripening. Physiologia Plantarum, Copenhagen, v.101, p. 727-739, 1997.

LOCKWOOD, D.; VINES, H. M. Red color enhancement of pimiento peppers with (2-chloroethyl) phosphonic acid. Journal of the American Society for Horticultural Science, Alexandria, v. 97, p. 192-197, 1972.

MAO, C.; MOTSENBOCKER, C.E. 2002. Effects of ethephon on Tabasco pepper fruit ripening and abscission at the fruitreceptacle junction. Scientia Horticulturae, Amsterdam, v.93, p.357-365, 2002

Mc LEOD, M.J.; ESHBAUGH, W.H.; GUTTMAN, S.I.; RAYLE, R.E.. An electrophoretic study of evolution in Capsicum (Solanaceae ). Evolution, Lawrence, v. 37, p. 562-574, 1983.

NIELSEN, T.; SKJAERBAEK, H.C.; KARLSEN, P. Carbohydrate metabolism during fruit development in sweet pepper (Capsicum annuum) plants. Physiologia Plantarum, Copenhagen, v. 82, p. 311-319, 1991.

PRETEL, M. T.; SERRANO, M.; AMOROS, A.; RIQUELME, F.; ROMAJORO, F. Non-involvement of ACC and ACC oxidase activity in pepper fruit ripening. Postharvest Biology and Technology, Amsterdam, v. 5, p. 295-302, 1995.

REIFSCHNEIDER, F.J.B. Capsicum Pimentas e Pimentões no Brasil. Brasília: Embrapa Comunicação para Transferência de Tecnologia/ Embrapa Hortaliças, 2000. 113p.

SALTVEIT JR, M. E. Carbon dioxide, ethylene, and color development in ripening mature green bell peppers. Journal of the American Society for Horticultural Science, Alexandria, v.120, p. 523-525, 1977.

SALTVEIT, M. E. Effect of ethylene on quality of fresh fruits and vegetables. Postharvest Biology and Technology, Amsterdam, v.15, p. 279-292, 1999.

TADESSE, T.; HEWETT, E. W.; NICHOLS, M. A.; FISHER, K.J. Changes in physiological attributes of sweet pepper $\mathrm{cV}$. Domino during fruit growth and development. Scientia Horticulturae, Amsterdam, v. 93, p. 91-103, 2002.

TUCKER, G. A. Introduction. In: SEYMOR, G. B.; TAYLOR, J. E.; TUCKER, G. A. Ed. Biochemisty of Fruit Ripening. Cambrige: Chapman \& Hall, 1993.

VILLAVINCENCIO, L E.; BLANKENSHIP, S.M.; SANDERS, D.C.; SWALLOW, W.H. Ethylene and carbon dioxide production in detached fruit of select pepper cultivars. Journal of the American Society for Horticultural Science, Alexandria, v.124, p.402-406, 1999

VILLAVINCENCIO, LE.; BLANKENSHIP, S.M.;SANDERS, D.C.; SWALLOW, W.H. Ethylene and carbon dioxide concentrations in attached fruits of pepper cultivars during ripening. Scientia Horticulturae, Amsterdam, v.91, p. 17-24, 2001. 